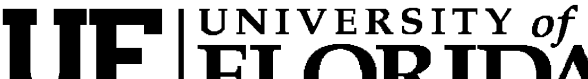 FLORIDA \\ IFAS Extension
}

\section{Chikungunya ${ }^{1}$}

\section{Roxanne Connelly, Christopher N. Mores, Chelsea T. Smartt, and Walter J. Tabachnick ${ }^{2}$ \\ What is Chikungunya? \\ What are the symptoms?}

Chikungunya is a virus that can be transmitted to humans by mosquitoes. The resulting illness is also called Chikungunya. It was originally described in the early 1950s after an outbreak in a Swahili village on the Makonde plateau that lies between Tanzania and Mozambique. The translation of Chikingunya from Makonde means "illness of the bended walker," "that which bends up," stooped over, walking bent over, or "bended walker." In India, it is known as Aakyda, meaning "stiff man" and Maakyda meaning "monkey-like". These words refer to the arthritic condition that occurs in some patients which gives rise to a stooped posture. The virus was first found in Asia, isolated in Bangkok, Thailand in 1958.

\section{The Virus}

Chikungunya virus, or CHIKV is a member of the Alphavirus genus in the family Togaviridae. CHIKV is related to other alphaviruses like Ross River, Sindbis and Venezuelan equine encephalitis viruses.
Reported symptoms include fever, chills, headache, rash and severe joint pain with or without swelling. Pain in the joints is a major feature of Chikungunya symptoms. The ankles and wrists are most commonly affected. Intense pain due to pressure placed on a wrist is commonly used to help diagnose the disease.

Symptoms begin to appear within $2-12$ days after being infected from the bite of an infected female mosquito. Initial symptoms include a sudden onset of flu-like symptoms including severe headache, chills, insomnia, fever, joint pain, nausea and vomiting. A rash may occur, first as a flush over the face and chest, followed by a rash that can have lesions. There can be mild hemorrhaging in children. Joint pain can persist for many months or even years after the other symptoms have subsided. All but a few patients recover.

\section{How is it transmitted to humans?}

The cycle of transmission is from mosquito to human and back to mosquito. Transmission to humans may occur when infected female mosquitoes

1. This document is ENY-736 (IN696) on mosquito tramsmitted disease, one of a series of the Entomology and Nematology Department, Florida Cooperative Extension Service, Institute of Food and Agricultural Sciences, University of Florida, Gainesville, FL. Publication date: April 2007. For more publications related to horticulture/agriculture, please visit the EDIS Website at http://edis.ifas.ufl.edu/.

2. C. Roxanne Connelly, associate professor, Christopher N. Mores and Chelsea T. Smartt, assistant professors, anc Walter J. Tabachnick, professor and director, Department of Entomology and Nematology, Florida Medical Entomology Laboratory, University of Florida, Vero Beach, FL. 
attempt to feed on a human host. The species of mosquitoes that may transmit the virus are Aedes aegypti (Fig. 1) and Aedes albopictus (Fig. 2). The eggs of these mosquitoes are laid just above the water line in water-holding containers. When the water level rises, the eggs hatch into larvae, where they continue to develop through the pupal stage, then to the adult that will fly to seek a blood meal. During the recent outbreak, a nurse caring for an infected patient in France came down with Chikungunya fever, suggesting that transmission may also occur from person to person, without the involvement of a mosquito vector.

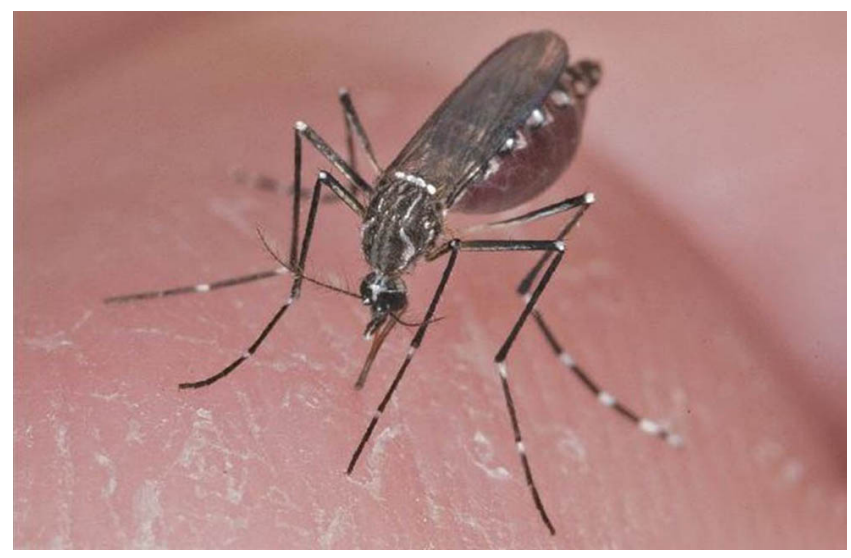

Figure 1. Aedes aegypti female. Credits: James M. Newman, UF/IFAS/FMEL

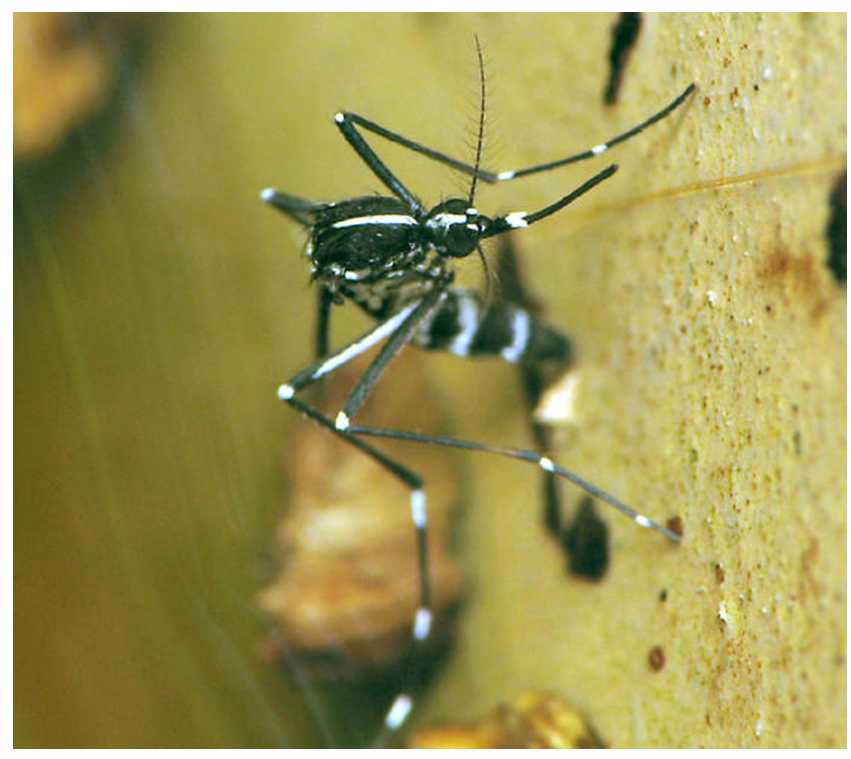

Figure 2. Aedes albopictus female. Credits: Sean McCann

\section{How is it diagnosed?}

Chikungunya is suspected when there is epidemic disease with the characteristic triad of symptoms of fever, rash and joint pain. Chikungunya can easily be confused with another mosquito-borne human pathogen called Dengue. The definitive diagnosis of Chikungunya requires laboratory testing. The virus can be detected during the first 48 hours of disease, and may be detected as late as day 4 in some patients. Usually Chikungunya is diagnosed by detecting patient antibodies produced by the immune response to the virus and found in the blood.

Antibodies to CHIKV persist in the blood in excess of 6 months.

\section{What is the cure?}

As with the other mosquito-borne viruses, there is no vaccine, nor is there a specific "cure." The fever and aches can be treated with traditional medicines like analgesics and anticonvulsants. Aspirin should be avoided. There is no medication that will kill the virus. Supportive care with rest is advised to deal with acute joint pain. Movement and mild exercise tend to improve stiffness. However, heavy exercise may exacerbate symptoms.

Chikungunya is usually self-limiting and recovery was thought to be universal, however, in January 2006, a high number of deaths (>200), either directly or indirectly related to infection with the virus, were reported from Reunion Island in the Indain Ocean. Excessive deaths were seen in patients who were 75 years of age or older. Once infected, immunity to further infection is long-lasting.

\section{Where does it occur?}

Chikungunya outbreaks have been reported from Africa and Southeast Asia. Most recently, between March 1, 2005 and April 30, 2006, there were 255,000 cases reported from Reunion Island in the Indian Ocean, with an estimated fatality rate of 1 per 1,000 cases. This was the largest Chikungunya outbreak ever described. The epidemic spread throughout the Indian Ocean, reaching India which had thousands of cases.

Infections may occur in areas that are not considered endemic, or where the virus does not normally occur. Travel and globalization increase the possibility of epidemic outbreaks in other regions 
around the globe, whenever the mosquitoes capable of transmitting CHIKV are found.

\section{Are Floridians at Risk?}

Transportation and globalization are important factors in assessing the risk of exposure to CHIKV for Floridians. The mosquito species that have been shown to be good vectors of CHIKV are present and well-established in Florida. It is believed that the CHIKV circulates at high levels in the blood of the human host, and therefore can directly infect capable mosquitoes. Travelers to endemic regions who become infected and then return to the US, or visitors to the US who are residents of those regions, may serve as a source of spreading the virus to local mosquitoes. This would initiate a transmission cycle in Florida capable of producing more cases and a potential Chikungunya epidemic.

\section{How can it be prevented?}

Surveillance. The current surveillance protocols for St. Louis Encephalitis virus (http://edis.ifas.ufl.edu/IN112) and West Nile virus (http://edis.ifas.ufl.edu/IN117) in Florida are not appropriate for Chikungunya. CHIKV is not transmitted to birds as is St. Louis and West Nile viruses. Then the first indication of the presence of CHIKV in Florida will be human cases. Mosquito control and public health agencies in Florida will need knowledge of Ae. aegypti and/or Ae. albopictus populations, where they are produced, and hot spots with large populations that could be easily targeted for control.

What can or should a mosquito control district do to control these populations? There is a great deal of information and experience clearly demonstrating that effective Ae. aegypti and Ae. albopictus control must involve public support and public participation to effectively reduce larval habitats. Plans must be in place on how to utilize citizens for these important surveillance and control efforts.

Prevent mosquito infection. It is clear that anyone with a Chikingunya infection should limit all exposure to mosquito bites. This can be done with use of repellents, or more appropriately but staying indoors or under mosquito netting. Doing so during the first $4-6$ days after infection when there is virus in the blood will avoid spreading the infection.

Reduce the mosquito vectors. Ae. albopictus and Ae. aegypti lay their eggs in containers to complete larval development. This includes containers from bucket-size to something as small as a bottle cap that holds water. Ae. albopictus in particular is able to utilize an array of both natural and artificial containers. To reduce the mosquito vectors, source reduction is the best method.

- Remove all container sources that can hold water including: bottles, cans, flower pots with saucer bases, clogged gutters, tires, lawn equipment such as wheel barrows and items that are covered with tarps.

- Other types of containers can be modified as follows: flush and replace water in bird baths and pet dishes several times during a week; cover water collection containers, such as rain barrels, with netting to prevent female mosquitoes from entering to lay eggs.

- Start a neighborhood watch program and sponsor container clean-up days to remove or modify water-holding containers in the area.

Be an informed traveler. Consult the Centers for Disease Control website for information on the current status of mosquito-borne disease transmission, as well as other diseases, across the globe prior to travel http://www.cdc.gov/travel/.

\section{References}

Josseran, L., C. Paquet, A. Zehgnoun, N. Caillere, A. Le Terte, J. Solet, and M. Ledrans. 2006. Chinungunya disease outbreak, Reunion Island. Emerging Infectious Diseases. 12:1994.

Parola, P., X. de-L., J. Jourdan, C. Rovery, V. Vaillant, P. Minodier, P. Broqui, A. Flahault, D. Raoult, and R. Charrel. 2006. Novel Chikungunya virus variant in travelers returning from Indian Ocean islands. Emerging Infectious Diseases. 12:1493-1498. 